\title{
Forecasting the Use of Institutional Elder Care in China: A System Dynamic Simulation
}

\author{
Rong Peng ${ }^{1, *}$ and Cathy Gong ${ }^{2}$ \\ ${ }^{1}$ National Economics Research Center, Guangdong University of Finance and Economics, Guangzhou, China \\ ${ }^{2}$ Centre for Research on Ageing, Health and Wellbeing, Australian National University, Canberra, Australia \\ ${ }^{*}$ Corresponding author
}

\begin{abstract}
With the rapid population aging in China, the older adults who needs for long-term care (LTC) increased dramatically in the past decades. To forecasting the use of institutional elder care in China, this study constructed a system dynamic model for LTC use to capture the decision-making process of living arrangement and institutional care use among the aging population in China. The results showed that the number of older adults living at institutions will increase from 200 million in 2015 to 290 million in 2035, which account for an increase of $45 \%$. This study provides policy implications that would assist policy makers understand the LTC delivery process and its influence factors, and help implement effective LTC policy scenarios.
\end{abstract}

Keywords-institutional care use; system dynamic simulation; long-term care

\section{INTRODUCTION}

Due to the rapid population aging, the number of Chinese elderly with activities daily living (ADL) limitation has been dramatically increased during the past decades. According to the fourth China urban and rural old population living conditions survey, China has more than 40.6 million ADL disabled elderly aged 60 and above in 2015, compared with the number of 22.6 million in 2000. The sharing of the elderly who need assistance in ADL among the total aging population double increased from 6.6 percent in 2000 to 18.3 percent in 2015. The health condition of disability resulting in great needs for long-term care (LTC) poses a serious challenge for caregiving in Chinese aging society.

Although the Chinese government has gradually established a national elder care policy during the past fifteen years, the problems existed in LTC delivery and financing system are still very tough. The policy suggests that home based care is the foundation for the LTC system, communitybased care provides a supportive resource for home-based care, and institutionalized care is a supplement. Under the traditional culture of filial piety, family members play a most important role in taking care of their old parents in China. It was reported that nearly 88 percent of Chinese older persons with disabilities are cared for by family members [1]. However, informal family care resources will be reduced in the future in terms of Chinese one-child policy, rural/urban migration and other societal changes. With the change of people's attitudes toward elder care and the increasing availability of institutional care facilities, nursing homes became a more and more popular option for many Chinese elderly adults and their families in recent years [2, 3].

\section{DYNAMIC MODEL FOR LTC USE}

System dynamics (SD) is a simulation modeling method used for representing the structure of complex systems and understanding their behavior over time [4]. Based on the identified problem, SD modeling firstly generates causal hypothesis and diagram causal relationship, and then translate qualitative hypothesis into quantitative simulation to test the policy influencing [5]. The patterns and trends resulting from simulation experimentation with different policies or strategies can be analyzed by modelers and stake holders to inform decision making. By capturing nonlinear phenomena and taking account the interdependence of competing sectors, SD models provide a more manageable and comprehensive way to project the future impact of policies on a system than do universal parameter estimation models.

This study firstly described the structure and function of the long-term care delivery system, and then constructed a dynamic model for this system. The LTC model simulated the living arrangement and LTC care use for the elderly living with at least one ADL limitation needing human assistance. The model simulates over a time frame of 20 years, from 2015 to 2035.

\section{A. Structure and Function of LTC Delivery System}

Figure I shows the structure and function of long-term care delivery system in China. This system consists of four agents, which are LTC demander, LTC supplier, government and social security department. The LTC demander in this study is limited to the elderly with at least one ADL limitation. They use LTC service provided by family, community or LTC facilities according to their care needs and ability to pay for the chargeable care service, as well as the accessibility of the care service. Government manages and monitors LTC service supply and utilization by implementing and adjusting policies. Social security system related to LTC is the public long-term care insurance, which is currently piloting in very few Chinese cities.

The agents in the LTC delivery system are interconnected and mutually acted on each other. For example, the agent of government can use the LTC policy as an instrument to adjust the institutional care provision by permitting more private capital invested institutions to enter the elder care market. The 
behavior of LTC utilization of the elderly could be affected by the initiate of public LTC insurance. In addition, the running of the LTC delivery system is also likely to be affected by external environment factors such as population aging process and endowment pension system. By dynamic matching the demand and supply of LTC, this system aims to reach the goal of meeting the LTC needs of the elderly, which is characterized as an important mission of population aging society.

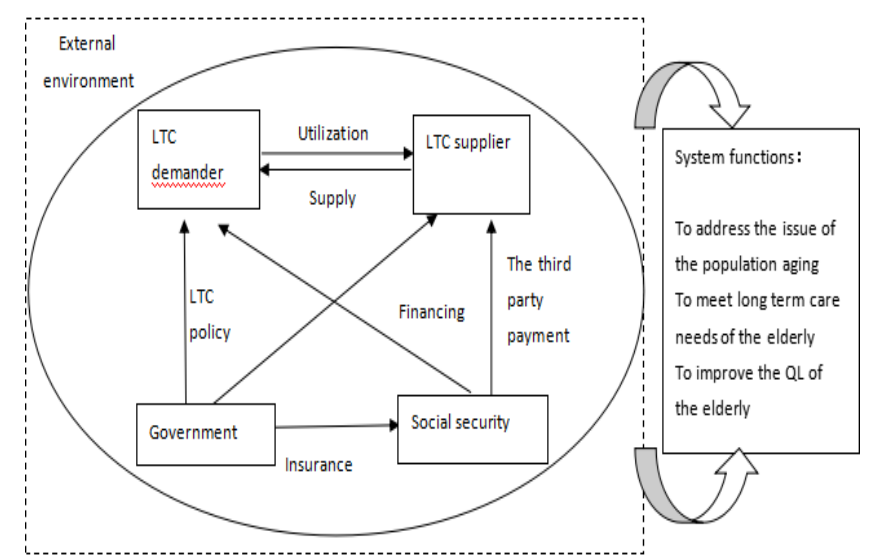

FIGURE I. STRUCTURE AND FUNCTION OF LONG-TERM CARE DELIVERY SYSTEM

\section{B. Stock-flow Diagram of LTC Delivery System}

Figure II represents the stock-flow diagram of LTC delivery system. In this system, the population of interest is comprised of elderly individuals aged 60 and above who have functional limitation that required human assistance. The LTC delivery system contains two subsystems: population subsystem and living arrangement subsystem. The two subsystem interact with each other through input and output variables contained in these subsystems and which are closely related.

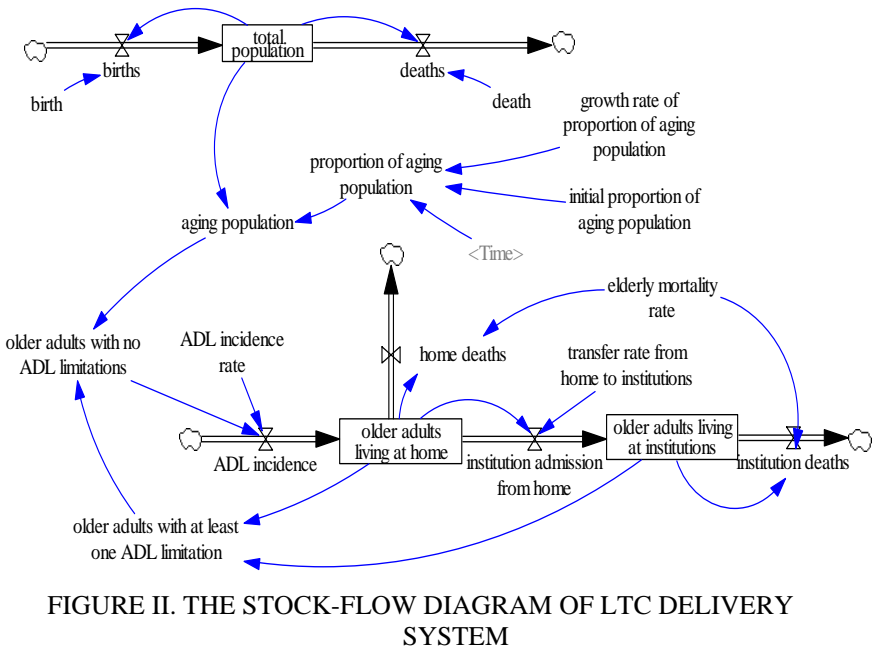

The population subsystem simulates the population aging process. The outcome variable is the number of the elderly population. The living arrangement subsystem simulates the living transition of the disabled elderly from home to institutions. The two output variables are the number of elders who live at home and the number of elders who live at institutions.

The main formulas involved in the model were listed below.

Total population= INTEG (births-deaths,1.37426e+009)

Births=total population*birth rate

Deaths=total population*death rate

Proportion of aging population=initial proportion of aging population*EXP(Time*growth rate of proportion of aging population)

Aging population=total population*proportion of aging population

Older adults with no ADL limitations=aging populationolder adults with at least one ADL limitation

Older adults with at least one ADL limitation=older adults living at home+older adults living at institutions

ADL incidence $=$ older adults with no ADL limitations*ADL incidence rate

Older adults living at home= INTEG (ADL incidence-home deaths-institution admission from home,3.86e+007)

Home deaths=older adults living at home*elderly mortality rate

Institution admission from home $=$ older adults living at home*transfer rate from home to institutions

Institution deaths=older adults living at institutions*elderly mortality rate

Older adults living at institutions $=$ INTEG (institution admission from home-institution deaths,2e+006)

\section{MODEL INPUTS AND PARAMETER ESTIMATION}

To start the simulation process, initial values need to be input into the system dynamic model, which runs during a time period of twenty years (from 2015 to 2035) in this study. Table I lists the initial values and data sources.

\section{TABLE I. MODEL INPUTS AND DATA SOURCES}

\begin{tabular}{|c|c|c|}
\hline Parameter & Value & Source \\
\hline Birth rate & 0.01207 & $\begin{array}{l}\text { China Statistical } \\
\text { Yearbook } 2016\end{array}$ \\
\hline Death rate & 0.00711 & $\begin{array}{l}\text { China Statistical } \\
\text { Yearbook } 2016\end{array}$ \\
\hline $\begin{array}{l}\text { Initial proportion of elderly } \\
\text { population }\end{array}$ & 0.161 & $\begin{array}{l}\text { China Statistical } \\
\text { Yearbook } 2016\end{array}$ \\
\hline Elderly mortality rate & 0.03215 & Census 2010 \\
\hline $\begin{array}{l}\text { Growth rate of proportion of } \\
\text { elderly population }\end{array}$ & 0.023 & Estimated by author \\
\hline ADL incidence rate & 0.012 & Estimated by author \\
\hline $\begin{array}{l}\text { Transition rate from home to } \\
\text { institutions }\end{array}$ & 0.0025 & Estimated by author \\
\hline
\end{tabular}

Demographic data were obtained from the China Statistical Yearbook 2016 and the Chinese Census 2010. The Growth rate of proportion of elderly population was estimated by using an 
exponential growth model based on cohort data of growth rate from 2000 to 2016.

The ADL incidence rate and transition rate from home to institutions were estimated by using data derived from the Chinese Longitudinal Healthy Longevity Survey (CLHLS), a large ongoing longitudinal study of Chinese elderly adults in longevity areas. CLHLS randomly selected half of the counties and cities in 22 provinces in China, representing $85 \%$ of the total population of the country. A detailed description of the sampling design and data quality has been reported elsewhere. The closed cohort of 2012 and 2014 waves of the CLHLS were used. The participant is considered disabled if he or she has at least one limitation in ADLs (bathing, dressing, eating, transferring, walking, and using the toilet). The elderly who live with family or alone are regarded as home living persons. The sample size of the community living elders aged 60 and above in the 2012 survey were 9415, among them 5900 survived at the follow-up survey, 758 were lost and 2757 died before the 2014 survey. Among the 5900 survived elders, 5743 provided living arrangement information at the 2014 survey, and 31 of them transferred from home to institutions between these two surveys. In the 2012 survey, the number of the community living elders without ADL limitation was 6788, among them 4890 survived at the 2014 survey. During these two surveys, 774 participants changed their health status of no ADL limitation to having at least one ADL limitation.

To estimate 1-year transition probabilities from two waves of survey conducted over a 2-year gap (2012 and 2014), the SPACE program was employed to model the dynamics of complex events [6]. The rules were that if the participants had the same state in both 2012 and 2014, then we assume they have been in that particular state in 2013 since there is no information on transition from the survey. If the participants were in different states in 2012 and 2014, then the transition is assumed to occur randomly between 2012 and 2014. A multinomial logistic regression models, with age and sex as covariates, were used to estimate the age-gender-specific transition rates. The total annual transition rates were calculated by weighting age and gender distribution on the age-genderspecific transition rates.

\section{MODEL VALIDATION}

To valid the model, one can check the values of the output variables of subsystems and compared them with data derived from public sources. In population system, the number of elderly population valued 2.21, 2.54, 2.92, 3.36 and 3.87 billion in 2015, 2020, 2025, 2030 and 2035 respectively. These five points were compared with the data published by United Nations [7]. One can see that the deviation between them was not significant-namely, between $0.45 \%$ and $6.05 \%$, which is within a reasonable range. In living option subsystem, the simulation value of the number of older adults with at least one ADL limitation were 45.34 million in 2020 and 58.34 million in 2030, while the official projected data suggested being 42.00 and 61.68 million respectively [8]. Thus, the model is considered validated and reasonable.

\section{THE NumBers OF EldERS USING INSTITUTIONAL CARE}

The number of older adults living at institution was showed in Figure III. It can be seen that the institutional care in China use will increase dramatically during the next 20 years. At the baseline case, the number of older adults living at institutions will increase from 200 million in 2015 to 290 million in 2035, which account for an increase of $45 \%$.

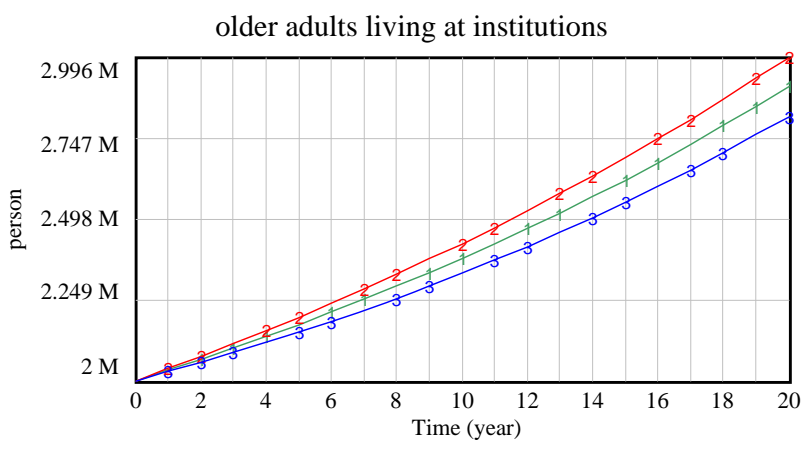

older adults living at institutions : $\begin{array}{rllllllll}-5 \% & 3 & 3 & 3 & 3 & 3 & 3 & 3 & 3\end{array}$ older adults living at institutions : $+5 \% 2$

older adults living at institutions : baseline

FIGURE III. THE PREDICTED NUMBER OF OLDER ADULTS LIVING AT INSTITUTION (2015-2035)

The predicted number of institutional care use was affected by transition rate from home to institutions. It can be seen from Figure III that the number of elder adults living at institutions will increase to 299.6 million in 2035 if the transition rate increase 5\%. The number will decrease to 290.4 million in 2035 if the transition rate is $95 \%$ of that at baseline. Thus, the use of institutional care use will change if the transition rates change during the period of twenty years.

\section{CONCLUSIONS}

This study provides a framework of China's LTC delivery system which synthesizes the demand of LTC, living options, and LTC service use. The results showed that the institutional care use in China will increase dramatically in the future. This study provides policy implications that would assist policy makers understand the LTC delivery process and its influence factors, and help implement effective LTC policy scenarios.

\section{ACKNOWLEDGMENT}

This work was supported by the National Natural Science Foundation of China (Grant number 71503054).

\section{REFERENCES}

[1] Su Q, Peng B, Chen J. An analysis on long-term care and influencing factors of the disabled elders: Based on rural-urban difference. Population \& Economics 2015, 25:69-76.

[2] Feng Z, Zhan HJ, Feng X, Liu C, Sun M, Mor V. An industry in the making: The emergence of institutional elder care in urban China. Journal of the American Geriatrics Society 2011, 59(4):738-744.

[3] Peng R, Wu B. Changes of health status and institutionalization among older adults in China. Journal of Aging and Health, 2015, 27(7):12231246.

[4] Marshall DA, Burgos-Liz L, Ijzerman MJ, Osgood ND, Padula WV, Higashi MK, Wong PK, Pasupathy KS, Crown W. Applying dynamic 
simulation modeling methods in health care delivery research-the SIMULATE checklist: Report of the ISPOR simulation modeling emerging good practices task force. Value in Health 2015, 18:5-16.

[5] Sterman J. System dynamics modeling: Tools for learning in a complex world. California Management Review 2001, 43:8-25.

[6] Cai L, Hayward MD, Lubitz J, Hagedorn A, Crimmins E. Estimation of multi-state life table functions and their variability from complex survey data using the SPACE Program. Demographic Research 2010, 22:129158.

[7] United Nations. United nations world population prospects: The 2015 revision. New York: United Nations (UN), 2015.

[8] Xinhua Network. China national committee on ageing: The number of Chinese elders with ADL limitation will be 42 million after 4 years. $2016 . \quad$ Available

http://m.zhongzhouw.com/news/shishiguancha/2016/1028/165306.html [accessed 12.04.2017]. 